\title{
Quasi-sure non-self-intersection for rough differential equations driven by fractional Brownian motion
}

\author{
Cheng Ouyang* $\quad$ William Roberson-Vickery $^{\dagger}$
}

\begin{abstract}
In this paper we study the self-intersection of paths solving elliptic stochastic differential equations driven by fractional Brownian motion. We show that such a path has no self-intersection - except for paths forming a set of zero $(r, q)$-capacity in the sample space - provided the dimension $d$ of the space and the Hurst parameter $H$ satisfy the inequality $d>r q+2 / H$. This inequality is sharp in the case of brownian motion and fractional brownian motion according to existing results. Various results exist for the critical case where $d=r q+4$ for Brownian motion.
\end{abstract}

Keywords: rough differential equations; stochastic differential equations; fractional Brownian motion; Malliavin calculus; capacity.

MSC2020 subject classifications: 60L20; 60H10; $60 \mathrm{H} 07$.

Submitted to ECP on November 2, 2021, final version accepted on February 12, 2022.

\section{Introduction}

The presence or absence as well as the number of self-intersections are important properties of the path of a stochastic process. These properties are studied along with differentiability vs. non-differentiability, modulus of continuity, and others (see e.g.[21][29]). Pólya [31] studied recurrence, the related property of whether a path returns to a point previously visited and how often, for paths of random walks. Lévy studied the question of recurrence and showed that in dimension 2 double points exist almost surely in [18][19]. Kakutani proved in 1944 that the paths of Brownian motion almost surely have no self-intersection for $d \geq 5$ [15]. This corresponds to $r=0$ and $H=1 / 2$ in the inequality $d>r q+2 / H$. Dvoretzky, Erdös, and Kakutani subsequently proved that there is no self intersection for Brownian motion for $d=4$, the critical dimension. Subsequently the authors proved that there are double intersections but no triple intersections in $d=3$, whereas there are intersections of arbitrary multiplicity for $d \leq 2$ [6][7][8].

Results on the capacity of the set of paths with self-intersection began with Fukushima, who showed in [10] that the set of Brownian motion paths which intersect themselves has zero $(1,2)$-capacity for $d \geq 7$. Takeda proved the result for Brownian Motion that the set of self-intersecting paths has zero $(r, q)$-capacity provided $d>r q+4$ [33]. Lyons proved that there is no self-intersection except on a set of zero $(1,2)$-capacity for the critical case $d=6$ [26]. Khoshnevisan reviews numerous results on the capacity of Brownian

${ }^{*}$ University of Illinois at Chicago, United States of America.

${ }^{\dagger}$ University of Illinois at Chicago, United States of America. E-mail: william. roberson. vickery@gmail.com 
self-intersection in the 2003 paper [16], where he also provides an elementary proof of past results and relates the self-intersection property to methods from the theory of renormalization groups. These authors use various definitions of capacity, resulting from the Ornstein-Uhlenbeck semi-group, the Sobolev norm for the Malliavin derivative, and the classical potential theory. These different definitions are mutually absolutely continuous as outer measures and comparable as norms [12][30][32].

We will use the definition of the capacity based on the Sobolev norm for the Malliavin deriviative in this paper. In $2018 \mathrm{Li}$ and Qian published a paper proving that selfintersecting paths of a fractional Brownian motion ( $\mathrm{fBm}$ ) with Hurst parameter $H$ form a set of zero $(r, q)$-capacity when $d>r q+2 / H$ [22], which our paper will show also applies to stochastic differential equations driven by $\mathrm{fBm}$. Li and Qian point out that the critical dimension case is currently an open problem for fractional Brownian motion because it is not possible to apply the classical potential theory. We follow the methods Boedihardjo et al. employed in [3] to analyze the self-intersection of the signature of a Brownian motion.

To employ the tools of the quasi-sure anlaysis we will have to show that the map between the sample space and solutions to the rough differential equation (RDE) is quasicontinuous. The results of Coutin and Qian demonstrate that dyadic interpolation of paths of fractional Brownian motion converge in p-variation except on a set of zero $(r, q)$ capacity in the sample space, provided the Hurst parameter $H$ satisfies $1 / 4<H<1$ and $p>1 / H$ [5]. These results were generalized and elaborated upon by both Boedihardjo et al. and Lyons and Qian [2][24]. Critically the dyadic interpolations do not converge in $p$ variation for any $p$ even almost surely for $H \leq 1 / 4$, so we will only consider $1 / 4<H<1$.

Combining this result with the universal limit theorem and a theorem of Malliavin we show that the map between the sample space and solutions to the RDE is quasicontinuous [25][27]. This allows us to apply the Chebyshev inequality for capacity to bound the capacity in a similar manner to the Chebyshev inequality for probability. We combine the Chebyshev inequality and bounds on the probability density for the path of the solution from Ouyang et al. in [20][1].

\section{Preliminaries}

\subsection{Fractional Brownian motion as a rough path}

Kolmogorov defined the process that Mandelbrot and Van Ness would later call fractional Brownian motion ( $\mathrm{fBm}$ ) in a 1940 paper [17] [28]. An $\mathrm{fBm}, B_{t}$, is a Gaussian stochastic process with mean zero and covariance

$$
\mathbb{E}\left[B_{t} B_{s}\right]=R(s, t):=\frac{1}{2}\left(s^{2 H}+t^{2 H}-|t-s|^{2 H}\right) .
$$

In order to analyze solutions to rough differential equations (rde) driven by such paths we apply Lyons' theory of rough paths [25]. Lyons introduced this theory in his seminal 1998 paper [23]. The context for the definition of a rough path on $\mathbb{R}^{d}$ is the tensor algebra

$$
T\left(\mathbb{R}^{d}\right)=\bigoplus_{n=0}^{\infty}\left(\mathbb{R}^{d}\right)^{\otimes n}
$$

where $\left(\mathbb{R}^{d}\right)^{\otimes 0}=\mathbb{R}$. The tensor algebra is equipped with a tensor product $\otimes$, and a rough path is a function $\mathbf{X}_{\mathbf{s}, \mathbf{t}}$ from the simplex $\Delta^{2}=\left\{(s, t) \in[0, T]^{2}: s<t\right\}$ to $T\left(\mathbb{R}^{d}\right)$, such that

$$
\mathbf{X}_{s, t}=\mathbf{X}_{s, u} \otimes \mathbf{X}_{u, t}
$$


for $s<u<t$. We refer to the $i$-th coordinate of $\mathbf{X}_{s, t}$ as $x_{s, t}^{i}$. The $p$-variation norm of a rough path can be defined

$$
\left\|\mathbf{X}_{s, t}\right\|_{p}=\max _{1 \leq i \leq\lfloor p\rfloor} \sup _{\mathcal{D} \subset[0, T]}\left(\sum_{l_{i} \in \mathcal{D}}\left\|x_{l_{i}, l_{i-1}}^{i}\right\|_{\left(\mathbb{R}^{d}\right)^{\otimes i}}^{\frac{i}{p}}\right)^{\frac{p}{i}},
$$

where $\lfloor p\rfloor$ denotes the integer floor, $\mathcal{D}$ a partition of $[0, T]$, and $\|\cdot\|_{\left(\mathbb{R}^{d}\right) \otimes i}$ the norm on the tensor product space induced by the Euclidean norm on $\mathbb{R}^{d}$. This is an example of a control, a super-additive function that plays to role of a norm in the rough path context [25].

For a path $X_{t}:[0, T] \rightarrow \mathbb{R}^{d}$ of bounded variation, where the Lebesgue-Stieltjes integral is defined, a canonical rough path representative exists that corresponds with the Chen signature,

$$
S\left(X_{t}\right)=\left(1, \int_{0 \leq s_{0} \leq t} d X_{s_{0}}, \int_{0 \leq s_{0} \leq s_{1} \leq t} d X_{s_{0}} \otimes d X_{s_{1}}, \ldots\right) .
$$

In [4] Chen proved - in the context of the algebraic topology - that the signature is a homomorphism between the space of paths and the tensor algebra. If we let $\gamma_{1}, \gamma_{2}$ be paths in $\mathbb{R}^{d}$ and let $*$ denote the concatenation operation, then

$$
S\left(\gamma_{1} * \gamma_{2}\right)=S\left(\gamma_{1}\right) \otimes S\left(\gamma_{2}\right) .
$$

This identity guarantees that $S\left(X_{t}\right)$ will be a multiplicative functional, i.e. a rough path in Lyons' definition [4]. The geometric $p$-rough paths, written $G \Omega^{p}\left(\mathbb{R}^{d}\right)$ is the completion in the $p$-variation norm of the space of signatures of paths of bounded variation.

Given a path $X_{t} \subset \mathbb{R}^{d}$ of bounded $p$-variation, we define the canonical lift to $G \Omega^{p}\left(\mathbb{R}^{d}\right)$ by approximating the path with dyadic interpolation. We define the $n$-th order dyadic interpolation of $X_{t}$ as the path coinciding with $X_{t}$ at the dyadic points $k / 2^{n}$ of the interval $[0, T]$, where $k$ is an integer, and piecewise linear in between. Explicitly,

$$
X_{t}^{(n)}=X_{\frac{k}{2^{n}}}+\left(t-\frac{k}{2^{n}}\right) \cdot 2^{-n} \cdot\left(X_{\frac{k+1}{2^{n}}}-X_{\frac{k}{2^{n}}}\right),
$$

for $t \in\left[\frac{k}{2^{n}}, \frac{k+1}{2^{n}}\right) \subset[0, T]$.

If the limit exists (in $p$-variation norm) we define

$$
\mathbf{X}_{0, t}=\lim _{n \rightarrow \infty} S\left(X_{t}^{(n)}\right) .
$$

If such a path exists it is called the canonical lift of $X_{t}$ because in general there are many distinct rough paths with the same increment $x_{s, t}^{1}$.

To summarize, the canonical lift can be defined using the following commutative diagram:

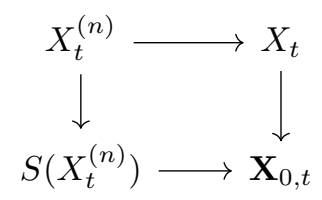

The top horizontal arrow represents convergence in $p$-variation norm for a path in $\mathbb{R}^{d}$, and the lower horizontal arrow represents convergence in $p$-variation norm for a rough 
path in $G \Omega^{p}\left(\mathbb{R}^{d}\right)$. The left vertical arrow indicates the Chen Signature map, which is only defined for paths of bounded variation.

When both the dyadic interpolations $X_{t}^{(n)}$ and the signatures $S\left(X_{t}^{(n)}\right)$ converge in $p$-variation norm in their respective spaces, we can define a "lift map" which maps $X_{t}$ to $\mathbf{X}_{0, t}$ and makes the diagram commute. This map is one to one since we can also map any rough path in $G \Omega^{p}\left(\mathbb{R}^{d}\right)$ to its increment $x_{0, t}^{1}$. We often identify the path $X_{t}$ with the rough path $\mathbf{X}_{0, t}$, and we will often throughout this paper refer only to the path $X_{t}$ while still applying the rough path technical machinery. In the case of fractional brownian motion - except for samples paths constituting a zero $(r, q)$-capacity or slim set - fBm has a canonical lift to the rough path space according to Coutin and Qian [5].

\subsection{Rough differential equations}

Lyons' monograph [25] develops the solution to a rough differential equation by building a theory of integration of one-forms defined on rough paths. It is too technical to develop the full theory here. It is sufficient to say that rough path integration with respect to Brownian motion is a generalization of Stratonovich's definition of the integral, and that it is possible to define integration pathwise and with respect to paths of bounded $p$-variation for $p>2$, where it is not possible to apply the Itô calculus. Lyons' Universal Limit Theorem proves that the Itô-Lyons solution map, which maps a driving path to the solution of an rde, $\Psi: G \Omega^{p}\left(\mathbb{R}^{d}\right) \rightarrow G \Omega^{p}\left(\mathbb{R}^{d}\right)$ is continuous in the $p$-variation norm.

Let $X_{t}$ be a $\mathrm{fBm}$, and let $V^{i}, 0 \leq i \leq d$ be a vector field satisfying elliptic conditions,

$$
v V(x) V^{*}(x) v^{*} \geq \lambda|v|^{2} \text { for } v, x \in \mathbb{R}^{d},
$$

where $V(x)=\left(V_{j}^{i}(x)\right)$, and $\lambda>0$.

The solution to the following rde

$$
Z_{t}=x_{0}+\int_{0}^{t} V_{0}\left(Z_{s}\right) d s+\sum_{i=1}^{d} \int_{0}^{t} V_{i}\left(Z_{s}\right) d X_{s}^{i}
$$

is then $\Psi(X)$.

We can extend the commutative diagram from the earlier section. We obtain a new diagram:

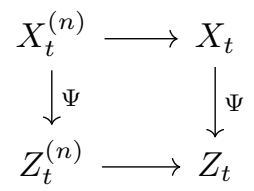

The Itô-Lyons map $\Psi$ sends the dyadic interpolation of the fBm $X_{t}^{(n)}$ to an approximation $Z_{t}^{(n)}$ of the solution $Z_{t}$. Both of these converge in $p$-variation, to the fBm $X_{t}$ and solution $Z_{t}$ respectively, because of the continuity of the solution map. This key fact will allow us to show that solutions to an rde driven by fBm are quasi-continuous.

\subsection{The Malliavin calculus and Malliavin derivative}

For some fixed $H \in\left(\frac{1}{4}, 1\right)$, we consider $(\Omega, \mathcal{F}, \mathbb{P})$ the canonical probability space associated with the fBm with Hurst parameter $H$. That is, $\Omega=\mathcal{C}_{0}([0, T])$ is the Banach space of continuous functions vanishing at 0 equipped with the supremum norm, $\mathcal{F}$ is the Borel sigma-algebra and $\mathbb{P}$ is the unique probability measure on $\Omega$ such that the 
canonical process $B=\left\{B_{t}=\left(B_{t}^{1}, \ldots, B_{t}^{d}\right), t \in[0, T]\right\}$ is a fractional Brownian motion with Hurst parameter $H$.

To situate fBm in the context of the Malliavin calculus let us define $R$ as follows,

$$
R(t, s):=\mathbb{E}\left[B_{s}^{j} B_{t}^{j}\right]=\frac{1}{2}\left(s^{2 H}+t^{2 H}-|t-s|^{2 H}\right), \quad \text { for } s, t \in[0,1] \text { and } j=1, \ldots, d \text {. }
$$

Malliavin techniques are essential in the analysis of $\mathrm{fBm}$, and we proceed here to introduce some of them (see [30] for further details): let $\mathcal{E}$ be the space of $\mathbb{R}^{d}$-valued step functions on $[0,1]$, and $\mathcal{H}$ the closure of $\mathcal{E}$ for the scalar product:

$$
\left\langle\left(\mathbf{1}_{\left[0, t_{1}\right]}, \cdots, \mathbf{1}_{\left[0, t_{d}\right]}\right),\left(\mathbf{1}_{\left[0, s_{1}\right]}, \cdots, \mathbf{1}_{\left[0, s_{d}\right]}\right)\right\rangle_{\mathcal{H}}=\sum_{i=1}^{d} R\left(t_{i}, s_{i}\right) .
$$

Some isometry arguments allow us to define the Wiener integral $W(h)=\int_{0}^{1}\left\langle h_{s}, d B_{s}\right\rangle$ for any element $h \in \mathcal{H}$, with the additional property $\mathbb{E}\left[W\left(h_{1}\right) W\left(h_{2}\right)\right]=\left\langle h_{1}, h_{2}\right\rangle_{\mathcal{H}}$ for any $h_{1}, h_{2} \in \mathcal{H}$.

An $\mathcal{F}$-measurable real valued random variable $F$ is then said to be cylindrical if it can be written, for a given $n \geq 1$, as

$$
F=f\left(W\left(h^{1}\right), \ldots, W\left(h^{n}\right)\right)=f\left(\int_{0}^{1}\left\langle h_{s}^{1}, d B_{s}\right\rangle, \ldots, \int_{0}^{1}\left\langle h_{s}^{n}, d B_{s}\right\rangle\right),
$$

where $h^{i} \in \mathcal{H}$ and $f: \mathbb{R}^{n} \rightarrow \mathbb{R}$ is a $C^{\infty}$ bounded function with bounded derivatives. The set of cylindrical random variables is denoted $\mathcal{S}$.

The Malliavin derivative is defined as follows: for $F \in \mathcal{S}$, the derivative of $F$ is the $\mathbb{R}^{d}$ valued stochastic process $\left(\mathbf{D}_{t} F\right)_{0 \leq t \leq 1}$ given by

$$
\mathbf{D}_{t} F=\sum_{i=1}^{n} h^{i}(t) \frac{\partial f}{\partial x_{i}}\left(W\left(h^{1}\right), \ldots, W\left(h^{n}\right)\right) .
$$

More generally, we can introduce iterated derivatives. If $F \in \mathcal{S}$, we set

$$
\mathbf{D}_{t_{1}, \ldots, t_{k}}^{k} F=\mathbf{D}_{t_{1}} \ldots \mathbf{D}_{t_{k}} F .
$$

For any $p \geq 1$, it can be checked that the operator $\mathbf{D}^{k}$ is closable from $\mathcal{S}$ into $\mathbf{L}^{p}\left(\Omega ; \mathcal{H}^{\otimes k}\right)$. We denote by $\mathbb{D}^{r, q}$ the closure of the class of cylindrical random variables with respect to the norm

$$
\|F\|_{r, q}=\left(\mathbb{E}\left(F^{q}\right)+\sum_{j=1}^{r} \mathbb{E}\left(\left\|\mathbf{D}^{j} F\right\|_{\mathcal{H}^{\otimes j}}^{q}\right)\right)^{\frac{1}{q}} .
$$

This norm is called the $(r, q)$-Sobolev norm.

\subsection{Capacity and quasi-continuity}

As mentioned in the introduction, there are various comparable definitions for capacity. For our purposes we will use the definition of capacity based on the $(r, q)$-Sobolev norm. Let $O$ be an open set with $O \subset \Omega$, the capacity is defined as follows,

$$
\operatorname{Cap}_{r, q}(O)=\inf \left\{\|F\|_{r, q}: F \in \mathbb{D}_{r, q}, F \geq 1 \text { on } O, F \geq 0, \mathbb{P} \text {-a.s. }\right\} .
$$

For a general set $\mathcal{A} \subset \Omega$

$$
\operatorname{Cap}_{r, q}(\mathcal{A})=\inf \left\{\operatorname{Cap}_{r, q}(O): O \text { open }, \mathcal{A} \subset O\right\} .
$$


Note that capacity is strictly increasing in $r$, and $r=0$ corresponds to the probability measure.

A random variable $F$, which is measurable with respect to the sigma-field $\mathcal{F}$, is $(r, q)$-quasi-continuous if for every $\epsilon>0$, there exists an open subset $\mathcal{O} \subset \Omega$ such that $\operatorname{Cap}_{r, q}(\mathcal{O})<\epsilon$ and $\left.F\right|_{\mathcal{O}^{c}}$ is continuous. Note that a norm must be specified on $\Omega$, which in our context is the supremum norm on $\Omega=\mathcal{C}_{0}([0, T])$. If $F$ is $(r, q)$-quasi-continuous for all $r, q \in \mathbb{N}, F$ is simply called quasi-continuous.

According to Theorem 2.3.3 in [27], every random variable $F \in \mathbb{D}_{r, q}$ has a quasicontinuous modification $F^{*}$ such that $F=F^{*}$ almost surely (a.s.) and $F^{*}$ is $(r, q)$-quasicontinuous. The $(r, q)$-quasi-continuous version $F^{*}$ is unique in the sense that $F^{*}=F^{*^{\prime}}$ except on a set of zero $(r, q)$-capacity if $F^{*^{\prime}}=F$ almost surely and $F^{*^{\prime}}$ is also $(r, q)$-quasicontinuous. Furthermore Theorem 2.3.5 from [27] gives us the following convergence result for what he calls the $(r, q)$-redefinition $F^{*}$,

Theorem 2.1. Given $\varphi_{n}, \varphi_{\infty} \in \mathbb{D}_{r, q}$, suppose $\left\|\varphi_{n}-\varphi_{\infty}\right\|_{r, q} \rightarrow 0$. Then it is possible to find a subsequence $n_{j}$ and a sequence of decreasing open sets $\mathcal{O}_{k}$ such that $\varphi_{n_{j}}^{*}$ converges uniformly towards $\varphi_{\infty}^{*}$ on $\mathcal{O}_{k}^{c}, c_{p, r}\left(\mathcal{O}_{k}\right) \rightarrow 0$, and $\mathcal{O}_{k}^{c}$ is compact.

Remark 2.2. Suppose $\varphi_{n}=\varphi_{n}^{*}$ and $\varphi_{n} \rightarrow \varphi_{\infty}$ quasi-surely. Then we have that $\varphi_{n_{j}} \rightarrow \varphi_{\infty}$ pointwise on each $\mathcal{O}_{k}^{c}$ except possibly on a zero $(r, q)$-capacity subset. Also from the theorem $\varphi_{n_{j}} \rightarrow \varphi_{\infty}^{*}$ pointwise on $\mathcal{O}_{k}^{c}$. We must then have $\varphi_{\infty}=\varphi_{\infty}^{*}$ on a full capacity subset of $\mathcal{O}_{k}^{c}$. Then taking the union of these sets we have that $\varphi_{\infty}=\varphi_{\infty}^{*}$ except on a set of zero $(r, q)$-capacity. In other words, $\varphi_{\infty}$ is its own $(r, q)$-redefinition and is thus $(r, q)$-quasi-continuous. This convergence theorem is the key technical tool applied in the following lemma.

We are now in a position to demonstrate that the solution $Z_{t}$ is quasi-continuous.

Lemma 2.3. The solution $Z_{t}$ to the stochastic rde (2.1) is quasi-continuous.

Proof. The map $\omega \mapsto X_{t}^{(n)}$ is quasi-continuous because it is interpolated at a finite number of points. The key idea is that the 1-variation only depends on the interpolation points, and it is bounded in terms of these values where interpolation occurs. $Z_{t}^{(n)}$ is also quasi-continuous because the 1 -variation of $Z_{t}^{(n)}$ is controlled by the 1-variation of $X_{t}^{(n)}$ due to the continuity of the solution map. According to Inahama in [14], $Z_{t}^{(n)}$ converges to $Z_{t}$ in $(r, q)$-Sobolev norm for all $r, q$ given the smoothness and boundedness conditions on the vector field. Finally we can apply the theorem and Remark 2.2 above with $\varphi_{n}=Z_{t}^{(n)}$ and $\varphi_{\infty}=Z_{t}$ to get the result.

\subsection{The Chebyshev inequality for capacity}

The reason we need to demonstrate quasi-continuity for the rde solution $Z_{t}$ is to apply the Chebyshev inequality for capacity,

$$
\operatorname{Cap}_{r, q}(|f|>R) \leq \frac{M_{r, q}|| f \|_{r, q}}{R}, R<0,
$$

One can find the proof of the inequality in Theorem 2.2 in II.iv.2.2 in [27]. The inequality is very similar to the classical Chebyshev inequality, and in the case of $r=0$ it reduces to the Chebyshev inequality. This inequality holds for any $(r, q)$-quasi-continuous function $f \in \mathbb{D}_{r}^{p}$, and $M_{r, q}$ only depends on $r$ and $q$.

\subsection{Sobolev type bounds on fBm driven SDE}

By a similar argument to [1, Lemma 4.1], we have the following bound on the Sobolev norm of the Malliavin derivative of the solution $Z_{t}$ to (2.1),

$$
\left\|\mathbb{D}\left(Z_{t}-Z_{s}\right)\right\|_{r, q}^{N} \leq C(t-s)^{N H} .
$$


Note that this implies that

$$
||\left|Z_{t}-Z_{s}\right|^{2 N}||_{r, q} \leq C|t-s|^{2 N H} .
$$

For an elliptic rde we know the density function exists, and we have the following bound on the density function from [20, Theorem 3.3]. Given $\epsilon>0$, we have for $\epsilon<s<t \leq T$ a constant $C$ which depends only on $\epsilon$ and $T$ such that

$$
p_{s, t}(y):=\mathbb{P}\left(Z_{t}-Z_{s} \in d y\right) \leq C(t-s)^{-d H} \exp \left(-\frac{|y|^{(2 H+1) \wedge 2}}{C(t-s)^{2 H}}\right) \leq C(t-s)^{-d H} .
$$

\subsection{Dyadic intervals}

The following theorem allows us to control the $p$-variation using dyadic partitions only, which is key to the proof of the main theorem here as well as in Kakutani's original paper [15].

Theorem 2.4. Let $\mathbf{X}=\left(X^{1}, \ldots, X^{\lfloor p\rfloor}\right)$ be a $p$-rough path.

(1) Given a constant $\gamma<p-1$, for $0 \leq s \leq t \leq 1$ and $1 \leq i \leq\lfloor p\rfloor$ define

$$
\rho_{i}(\mathbf{X}, s, t)=\sum_{m=1}^{\infty} m^{\gamma} \sum_{k=1}^{2^{m}}\left|X_{t_{m}^{k-1}, t_{m}^{k}}^{i}\right|^{\frac{p}{i}}
$$

where $\left(t_{m}^{k}\right)_{0 \leq k \leq 2^{m}}$ is the dyadic partition of $[s, t]$. Then there exists a constant $C=C(p, \gamma)$, such that

$$
\sup _{\mathcal{P}([s, t])} \sum_{l}\left|X_{t_{l-1}, t_{l}}^{i}\right|^{\frac{p}{i}} \leq C(p, \gamma) \sum_{j=1}^{i} \rho_{j}(\mathbf{X}, s, t)
$$

for all $1 \leq i \leq\lfloor p\rfloor$ and $0 \leq s \leq t \leq 1$, where the supremum is taken over all finite partitions of $[s, t]$.

Refer to [24] for the proof.

\section{Main theorem}

This brings us to the statement of our main theorem which is consistent with previous results such as [15] in the case of $r=0$ and $H=\frac{1}{2}$ or [3] for $H=\frac{1}{2}$.

Theorem 3.1. Let $H>\frac{1}{4}$, and let $Z_{t}$ denote the solution to a d-dimensional stochastic differential equation of the form (2.1) driven by a fractional Brownian motion, then

$$
\operatorname{Cap}_{(r, q)}\left(\left\{Z_{t}=Z_{s} \text { for } 0 \leq s<t \leq T\right\}\right)=0
$$

if

$$
\frac{2}{H}+r q<d
$$

We divide the proof of the main theorem into three sections. First we bound the capacity of the event that the increment maximum exceeds $\eta$ (bounding increment above).

\subsection{Bounding capacity of magnitude of increment}

Proposition 3.2. Let $\eta>0$, then for $N>r$ satisfying $\frac{N}{H}$ is an even integer,

$$
\operatorname{Cap}_{(r, q)}\left(\max _{t_{0} \leq t \leq t_{1}}\left|Z_{t}-Z_{t_{0}}\right|>\eta\right) \leq C \eta^{-\frac{N}{H}}\left|t_{1}-t_{0}\right|^{N}
$$

where $C$ depends on $N, q, r, d$. 
Proof. Let $Z_{t}$ be the solution to equation (2.1). Choose $\lambda>0$ so that $\eta>\lambda^{1 / p}$, and let $\omega(s, t)$ denote the $p$-variation for $p>\frac{1}{H}$. Let $\rho_{1}, C(p, \gamma)$ denote the constants in theorem 2.4. Then for $C=C(p, \gamma)^{-1}$, we have

$$
\left\{\max _{t_{0} \leq t \leq t_{1}}\left|Z_{t}-Z_{t_{0}}\right|>\eta\right\} \subseteq\left\{\omega\left(t_{0}, t_{1}\right)>\lambda\right\} \subseteq\left\{\rho_{1}>C \lambda\right\} .
$$

Given $\theta>0$ let $C_{\theta}>0$ satisfy

$$
C_{\theta} \sum_{m=1}^{\infty} m^{\gamma} 2^{-m \theta} \leq C
$$

Then we have $\rho_{1}>C \lambda$ implies

$$
\rho_{1}=\sum_{m=1}^{\infty} m^{\gamma} \sum_{k=1}^{2^{m}}\left|Z_{t_{m}^{k}}-Z_{t_{m}^{k-1}}\right|^{p}>C \lambda \geq \sum_{m=1}^{\infty} C_{\theta} \lambda m^{\gamma} 2^{-m \theta}
$$

where the $t_{m}^{k}$ are the points of the dyadic partition defined in theorem 2.4.

If the inequality above holds, then for some $m$ we must have

$$
\sum_{k=1}^{2^{m}}\left|Z_{t_{m}^{k}}-Z_{t_{m}^{k-1}}\right|^{p}>C_{\theta} \lambda 2^{-m \theta}=\sum_{k=1}^{2^{m}} C_{\theta} \lambda 2^{-m(\theta+1)} .
$$

From this inequality it follows that for some term indexed by $k$,

$$
\left|Z_{t_{m}^{k}}-Z_{t_{m}^{k-1}}\right|^{p}>C_{\theta} \lambda 2^{-m(\theta+1)} .
$$

Accordingly

$$
\left\{\rho_{1}>C \lambda\right\} \subseteq \bigcup_{m=1}^{\infty} \bigcup_{k=1}^{2^{m}}\left\{\left|Z_{t_{m}^{k}}-Z_{t_{m}^{k-1}}\right|^{p}>C_{\theta} \lambda 2^{-m(\theta+1)}\right\} .
$$

It follows from the sub-additivity of capacity that

$$
\operatorname{Cap}_{r, q}\left(\left\{\rho_{1}>C \lambda\right\}\right) \leq \sum_{m=1}^{\infty} \sum_{k=1}^{2^{m}} \operatorname{Cap}_{r, q}\left(\left\{\left|Z_{t_{m}^{k}}-Z_{t_{m}^{k-1}}\right|^{p}>C_{\theta} \lambda 2^{-m(\theta+1)}\right\}\right) .
$$

As explained in the introduction, $Z_{t}$ is quasi-continuous. To apply the Chebyshev inequality we need to replace $\left|Z_{t}-Z_{s}\right|^{p}$ with $\left|Z_{t}-Z_{s}\right|^{\frac{N}{H}}$, where $\frac{N}{H}$ is an even integer greater than $p$, since composition with a smooth function preserves quasi-continuity. We can then apply the Chebyshev inequality for capacity and inequality (2.2) to yield

$$
\begin{aligned}
\operatorname{Cap}_{r, q} & \left(\left\{\left|Z_{t_{m}^{k}}-Z_{t_{m}^{k-1}}\right|^{N / H}>\left(C_{\theta} \lambda\right)^{N / H p} 2^{-m N(\theta+1) / H p}\right\}\right) \\
& \leq \frac{M}{\left(C_{\theta} \lambda\right)^{N / H p}}|| Z_{t_{m}^{k}}-\left.Z_{t_{m}^{k-1}}\right|^{N / H} \|_{r, q} 2^{m N(\theta+1) / H p} \\
& \leq \frac{\tilde{M}}{\left(C_{\theta} \lambda\right)^{N / H p}}\left(t_{m}^{k}-t_{m}^{k-1}\right)^{N} 2^{m N(\theta+1) / H p} \\
& =\frac{\tilde{M}\left|t_{1}-t_{0}\right|^{N}}{\left(C_{\theta} \lambda\right)^{N / H p}} 2^{m N\left(\frac{(\theta+1)}{H p}-1\right)}
\end{aligned}
$$

From this it follows that

$$
\operatorname{Cap}_{(r, q)}\left(\max _{t_{0} \leq t \leq t_{1}}\left|Z_{t}-Z_{t_{0}}\right|>\eta\right) \leq \frac{\tilde{M}\left|t_{1}-t_{0}\right|^{N}}{\left(C_{\theta} \lambda\right)^{N / H p}} \sum_{m=1}^{\infty} 2^{m\left(N\left(\frac{(\theta+1)}{H p}-1\right)+1\right)}
$$


Quasi-sure non-self-intersection for rough differential equations

So we need to have

$$
N\left(\frac{(\theta+1)}{H p}-1\right)+1<0
$$

for the sum to converge to a finite number. This is possible since $p>\frac{1}{H}$. Finally we obtain

$$
\operatorname{Cap}_{(r, q)}\left(\max _{t_{0} \leq t \leq t_{1}}\left|Z_{t}-Z_{t_{0}}\right|>\eta\right) \leq C_{N, q, d} \frac{\left|t_{1}-t_{0}\right|^{N}}{\lambda^{N / H p}} \leq C_{N, q, d} \frac{\left|t_{1}-t_{0}\right|^{N}}{\eta^{N / H}}
$$

\subsection{Bounding capacity of magnitude of total displacement}

We now need bounds on the capacity of the event that the increment is smaller than $\eta$.

Proposition 3.3. Let $\tau>0$ and $Z_{t}$ denote the solution to rde (2.1) as before, then

$$
\operatorname{Cap}_{(r, q)}\left(\left|Z_{t_{1}}-Z_{t_{0}}\right| \leq \eta\right) \leq \frac{C}{\left|t_{1}-t_{0}\right|^{\frac{d H}{\tau q}}} \eta^{\frac{d}{\tau q}-r}
$$

Proof. Define a function $f \in C^{\infty}\left(\mathbb{R}^{d}\right)$ such that

$$
\begin{cases}0 \leq f(x) \leq 1 & \text { for all } x \in \mathbb{R}^{d} \\ f(x)=1 & \text { for }|x| \leq \eta \\ f(x)=0 & \text { for }|x| \geq 2 \eta \\ \left|\nabla^{k} f(x)\right| \leq \frac{C_{n}}{\eta^{k}} & \text { for all } x \in \mathbb{R}^{d}\end{cases}
$$

Define $F(\omega, t)=f\left(Z_{t}-Z_{t_{0}}\right)$. Using the chain rule and (2.2) we can conclude that for all $q^{\prime}>1$,

$$
\|F\|_{r, q^{\prime}} \leq \frac{C\left(r, q^{\prime}, n, d\right)}{\eta^{r}}
$$

We can apply the Chebyshev inequality for capacity because $F$ is smooth and $Z_{t}$ is quasi-continuous, so their composition is quasi-continuous. Let $\tau>1$, then

$$
\begin{aligned}
\operatorname{Cap}_{r, q}\left(\left|Z_{t_{1}}-Z_{t_{0}}\right| \leq \eta\right) & \leq \operatorname{Cap}_{r, q}(|F| \geq 1) \\
& \leq C_{r, q}|| F \|_{r, q} \\
& \leq C_{r, q} \sum_{i=0}^{r} \mathbb{E}\left(\left|D^{i} F\right|^{q} \mathbf{1}_{\left\{\left|Z_{t_{1}}-Z_{t_{0}}\right| \leq 2 \eta\right\}}\right)^{1 / q} \\
& \leq\|F\|_{r, q_{1}} \mathbb{P}\left(\left|Z_{t_{1}}-Z_{t_{0}}\right| \leq 2 \eta\right)^{1 / \tau q} \\
& \leq \frac{C}{\eta^{r}} \mathbb{P}\left(\left|Z_{t_{1}}-Z_{t_{0}}\right| \leq 2 \eta\right)^{1 / \tau q}
\end{aligned}
$$

where $q_{1}=\frac{\tau q}{(\tau-1)}$. We can use the bound (2.3) for density of an elliptic stochastic pde. This is the reason why we require the vector field $V_{i}$ in (2.1) to be elliptic (it is possible that we can loosen this condition to hypoellipticity). Given (2.3), we obtain

$$
\begin{aligned}
\operatorname{Cap}_{r, q}\left(\left|Z_{t_{1}}-Z_{t_{0}}\right| \leq \eta\right) & \leq \frac{C}{\eta^{r}} \mathbb{P}\left(\left|Z_{t_{1}}-Z_{t_{0}}\right| \leq 2 \eta\right)^{1 / \tau q} \\
& \leq \frac{C}{\eta^{r}}\left(\int \mathbf{1}_{\{|x| \leq 2 \eta\}} p_{t_{1}-t_{0}}(x) d x\right)^{1 / \tau q} \\
& \leq \frac{C}{\eta^{r}}\left(\int \mathbf{1}_{\{|x| \leq 2 \eta\}} c_{1} t^{-d H} \exp \left(-\frac{|y-x|^{2 H+1 \wedge 2}}{c_{2} t^{2 H}}\right) d x\right)^{1 / \tau q} \\
& \leq \frac{C^{\prime}}{\eta^{r}\left|t_{1}-t_{0}\right| d H / \tau q}\left(\int \mathbf{1}_{\{|x| \leq 2 \eta\}} d x\right)^{1 / \tau q} \\
& \leq \frac{C^{\prime}}{\left|t_{1}-t_{0}\right|^{d H / \tau q}} \eta^{\frac{d}{\tau q}-r}
\end{aligned}
$$

which proves the result. 


\subsection{Combining bounds to prove main theorem}

Finally we combine the two bounds for the capacity of events related to the increment size. We subdivide the intervals where a self-intersection might occur into dyadic subintervals in order to use these controls on capacity to estimate the capacity of the self-intersection event.

Proof. Let $\left[s_{0}, s_{1}\right]$ and $\left[t_{0}, t_{1}\right]$ be dyadic intervals. Observe that for $s \in\left[s_{0}, s_{1}\right]$ and $t \in$ $\left[t_{0}, t_{1}\right]$, if $Z_{s}=Z_{t}$, then

$$
\left|Z_{t_{0}}-Z_{s_{0}}\right|=\left|Z_{t_{0}}-Z_{t}+Z_{s}-Z_{s_{0}}\right| \leq\left|Z_{t_{0}}-Z_{t}\right|+\left|Z_{s}-Z_{s_{0}}\right|
$$

Accordingly,

$$
\begin{aligned}
& \left\{Z_{t}=Z_{s}: \text { for some } s \in\left[s_{0}, s_{1}\right] \text { and } t \in\left[t_{0}, t_{1}\right]\right\} \\
& \qquad \subseteq\left\{\left|Z_{t_{0}}-Z_{s_{0}}\right| \leq 2 \eta\right\} \bigcup\left\{\max _{t \in\left[t_{0}, t_{1}\right]}\left|Z_{t}-Z_{t_{0}}\right|>\eta\right\} \bigcup\left\{\max _{s \in\left[s_{0}, s_{1}\right]}\left|Z_{s}-Z_{s_{0}}\right|>\eta\right\} .
\end{aligned}
$$

Note that the $q$-th power of the capacity is subadditive due to the Meyer's inequality and the integral representation formula [2]. Without loss of generality assume $\left|t_{1}-t_{0}\right|=$ $\left|s_{1}-s_{0}\right|=\Delta$. Then we obtain,

$$
\begin{aligned}
\operatorname{Cap}_{r, q}\left(\left\{Z_{t}=Z_{s}\right.\right. & \left.\left.: \text { for some } s \in\left[s_{0}, s_{1}\right] \text { and } t \in\left[t_{0}, t_{1}\right]\right\}\right)^{q} \\
& \leq \operatorname{Cap}_{r, q}\left(\left\{\left|Z_{t_{0}}-Z_{s_{0}}\right| \leq 2 \eta\right\}\right)^{q} \\
& +\operatorname{Cap}_{r, q}\left(\left\{\max _{t \in\left[t_{0}, t_{1}\right]}\left|Z_{t}-Z_{t_{0}}\right|>\eta\right\}\right)^{q} \\
& +\operatorname{Cap}_{r, q}\left(\left\{\max _{s \in\left[s_{0}, s_{1}\right]}\left|Z_{s}-Z_{s_{0}}\right|>\eta\right\}\right)^{q} \\
& \leq \frac{C}{\left|t_{0}-s_{0}\right|^{\frac{d H}{\tau}}} \eta^{\frac{d}{\tau}-r q}+C \eta^{-\frac{N q}{H}}\left|t_{1}-t_{0}\right|^{N q}+C \eta^{-\frac{N q}{H}}\left|s_{1}-s_{0}\right|^{N q} \\
& =\frac{C}{\left|t_{0}-s_{0}\right|^{\frac{d H}{\tau}}} \eta^{\frac{d}{\tau}-r q}+C \eta^{-\frac{N q}{H}} \Delta^{N q}+C \eta^{-\frac{N q}{H}} \Delta^{N q} .
\end{aligned}
$$

Now consider covering the intervals $\left[s_{0}, s_{1}\right]$ and $\left[t_{0}, t_{1}\right]$ with smaller dyadic intervals of length $\Delta 2^{-l}$. Any two intervals of the form $\left[s_{0}+k 2^{-l}, s_{0}+(k+1) 2^{-l}\right]$ and $\left[t_{0}+(j+\right.$ 1) $\left.2^{-l}, t_{1}+(j+1) 2^{-l}\right]$ are separated by at least a distance of $\left|t_{0}-s_{1}\right|$. There are also $2^{2 l}$ possible pairs of the new intervals that could contain the self-intersection. This yields

$$
\begin{aligned}
\operatorname{Cap}_{r, q}\left(\left\{Z_{t}=Z_{s}: \quad s \in\left[s_{0}, s_{1}\right] \text { and } s \in\left[t_{0}, t_{1}\right]\right\}\right)^{q} \\
\leq 2^{2 l}\left(\frac{C}{\left|t_{0}-s_{1}\right|^{\frac{d H}{\tau}}} \eta^{\frac{d}{\tau}-r q}+C \eta^{-\frac{N q}{H}}\left(\Delta 2^{-l}\right)^{N q}+C \eta^{-\frac{N q}{H}}\left(\Delta 2^{-l}\right)^{N q}\right) \\
\leq C 2^{2 l} \eta^{\frac{d}{\tau}-r q}+C^{\prime} \eta^{-\frac{N q}{H}} 2^{-l(N q-2)+1} .
\end{aligned}
$$

Now let $\eta=2^{-\sigma l}$ approach zero.

$$
\begin{aligned}
\operatorname{Cap}_{r, q}\left(\left\{Z_{t}=Z_{s}: \quad\right.\right. & \left.\left.s \in\left[s_{0}, s_{1}\right] \text { and } s \in\left[t_{0}, t_{1}\right]\right\}\right)^{q} \\
& \leq C 2^{-l\left(\sigma\left(\frac{d}{\tau}-r q\right)-2\right)}+C^{\prime} 2^{-l\left(N q\left(\frac{\sigma}{H}+1\right)-2\right)+1}
\end{aligned}
$$

In order for this to converge to zero we need

$$
\sigma\left(\frac{d}{\tau}-r q\right)-2>0
$$


and

$$
\frac{\sigma}{H}+1>0 .
$$

There is a sigma satisfying both these inequalities if

$$
\frac{2}{\frac{d}{\tau}-r q}<\sigma<H
$$

This is possible if

$$
\frac{2}{H}+r q<d
$$

since $\tau>1$.

\section{Conclusion}

The critical case remains an open question for both fractional Brownian motion and stochastic differential equations driven by fractional Brownian motion. The difficulty to extend our result to solutions to hypo-elliptic stochastic differential equations stems from the lack of estimate (2.3). A key to obtain such an estimate is a good control of the Malliavin matrix of $Z_{t}-Z_{s}$, which is not an easy task for hypo-elliptic equations.

\section{References}

[1] F. Baudoin, E. Nualart, E. C. Ouyang, and S. Tindel, On probability laws of solutions to differential systems Driven by a fractional Brownian motion, Ann. Probab. 44(4): 2554-2590, 2016. MR3531675

[2] H. Boedihardjo, X. Geng and Z. Qian, Quasi-sure existence of Gaussian rough paths and large deviation principles for capacities, Osaka J. Math. 53(4): 941-970. 2016. MR3554850

[3] H. Boedihardjo, X. Geng, X. Liu, Z. Qian, A Quasi-sure Non-degeneracy Property for the Brownian Rough Path, Potential Analysis. volume 51, 2019. MR3981439

[4] K.T. Chen, Integration of paths - a faithful representation of paths by non-commutative formal power series. Trans. Am. Math. Soc., 89:395-407, 1958. MR0106258

[5] L. Coutin and Z. Qian, Stochastic analysis, rough path analysis and fractional Brownian motions, Probab. Theory Relat. Fields 122 (1), 108-140, 2002. MR1883719

[6] A. Dvoretzky, P. Erdős, and S. Kakutani, Double points of paths of Brownian motion in n-space, Acta Sci. Math. Szeged 12: 75-81, 1950. MR0034972

[7] A. Dvoretzky, P. Erdös, and S. Kakutani, Multiple points of paths of Brownian motion in the plane. Bull. Res. Council Israel 3, 364-371, 1954. MR0067402

[8] A. Dvoretzky, P. Erdös, S. Kakutani, and S. J. Taylor, Triple points of Brownian paths in 3-space. Proc. Cambridge Philos. Soc. 53, 856-862 (1957). MR0094855

[9] P. K. Friz and N. B. Victoir, Multidimensional stochastic processes as rough paths, Cambridge University Press, Cambridge, 2010. MR2604669

[10] M. Fukushima, Basic properties of Brownian motion and a capacity on the Wiener space, J. Math. Soc. Japan 36 (1): 161-176, 1984. MR0723601

[11] M. Fukushima, Y. Oshima, and M. Takeda, Dirichlet Forms and Symmetric Markov Processes, De Gruyter, Berlin/New York 2010. MR2778606

[12] M. Hinz and S. Kang, Capacities, Removable Sets and $L^{p}$-Uniqueness on Wiener Spaces, Potential Analysis 54:503-533, 2021. MR4215349

[13] Y. Inahama, Quasi-Sure Existence of Brownian Rough Paths and a Construction of Brownian Pants, Infin. Dimens. Anal. Quantum Probab. Relat. Top. 9 (4): 513-528, 2006. MR2282717

[14] Y. Inahama, Malliavin Differentiability of Solutions of Rough Differential Equations. Preprint. arXiv:1312.7621 MR3229800

[15] S. Kakutani, On Brownian Motions in n-Space, Proceedings of the Imperial Academy 20 (9): 648-652, 1944. MR0014646 
Quasi-sure non-self-intersection for rough differential equations

[16] D. Khoshnevisan, Intersections of Brownian Motion. Expo. Math. 21: 97-114, 2003. MR1978059

[17] A.N. Kolmogorov, Wienersche spiralen und einige andere interessante Kurven in Hilbertscen Raum, C. R. (Dokl.) Acad. Sci. URSS (NS) 26, 115-118, 1940. MR0003441

[18] P. Lévy, Les mouvements browniens plans, Amer. Journ. of Math., 62, 1940. MR0002734

[19] P. Lévy, Processus Stochastiques et Mouvement Brownien, Gauthier-Villars, Paris, 1948. MR0190953

[20] S. Lou and C. Ouyang, Fractal dimensions of rough differential equations driven by fractional Brownian motions. Stochastic Processes and their Applications. Volume 126, Issue 8, 24102429, 2016. MR3505232

[21] I. Karatzas and S. Shreve, Brownian motion and stochastic calculus, volume 113. Springer Science \& Business Media, 2012. MR3184878

[22] J. Li and Z. Qian, Fine properties of fractional Brownian motions on Wiener space, Journal of Mathematical Analysis and Applications. Volume 473, Issue 1, 1, Pages 1411-173, 2019. MR3912816

[23] T. Lyons, Differential equations driven by rough signals, Rev. Mat. Iberoamericana 14 (2), 215-310, 1998. MR1654527

[24] T. Lyons and Z. Qian, System control and rough paths, Oxford University Press, New York, 2002. MR2036784

[25] T. Lyons, M. Caruana, and T. Lévy, Differential Equations Driven by Rough Paths, Springer, Berlin, 2007. MR2314753

[26] T. Lyons, The critical dimension at which quasi-every Brownian path is self-avoiding, Adv. in Appl. Probab. 18: 87-99, 1986. MR0868510

[27] P. Malliavin, Stochastic analysis, Springer, Berlin, 1997. MR1450093

[28] B.B. Mandelbrot and J.W. Van Ness, Fractional Brownian motions, fractional noises and applications, SIAM Rev. 10 (4) (1968) 422-437. MR0242239

[29] P. Mörters and Y. Peres, Brownian Motion, Cambridge University Press, Cambridge, 2010. MR2604525

[30] D. Nualart, The Malliavin Calculus and Related Topics, Springer, 2006. MR2200233

[31] G. Pólya, Über eine Aufgabe der Wahrscheinlichkeitsrechnung betreffend die Irrfahrt im Strassensatz, Math. Ann. 84, 149-160 MR1512028

[32] H. Sugita, Positive generalized Wiener functions and potential theory over abstract Wiener spaces, Osaka J. Math. 25: 665-696, 1988. MR0969026

[33] M. Takeda, (r, p)-Capacity on the Wiener space and properties of Brownian motion, Z. Wahrscheinlichkeitstheorie verw. Gebiete 68: 149-162, 1984. MR0767798 